\title{
Hypnotizability Dependent Autonomic Modulation during a Low Attentional Task
}

\author{
R Balocchi ${ }^{1}$, G Paoletti $^{2}$, EL Santarcangelo ${ }^{3}$, E Scattina $^{3}$, \\ L Sebastiani $^{3}$, A Macerata ${ }^{1,3}$, M Varanini $^{1}$ \\ ${ }^{1}$ Institute of Clinical Physiology, National Research Council, Pisa, Italy \\ ${ }^{2}$ Department of Physiology, University of Siena, Siena, Italy \\ ${ }^{3}$ Department of Human Physiology, University of Pisa, Pisa, Italy
}

\begin{abstract}
Hypnotizability-dependent changes in heart rate and blood pressure in the time domain were studied during low attentional tasks in subjects with high or low hypnotic susceptibility watching a relaxing and an alerting movie. All participants reported relaxation during the former, while only part of them was stressed by the latter. During relaxation hypnotizability did not modulate mean heart rate and blood pressure, but affected the variability of the latter, which indirectly indicates differences in the autonomic pattern associated with the low attentional relaxing movie with respect to simple relaxation and highlighted the hypnotizabilityrelated role of the emotional content associated with a relaxation response. In line with previous studies the results suggested a role for hypnotizability in the control of the vascular resistance.
\end{abstract}

\section{Introduction}

Hypnotic susceptibility is a cognitive trait responsible for the possibility to accept suggestions and modulate perception and behaviour accordingly [1]. It can be measured through scales and has its anatomo-functional basis in the supervisory attentional system, located in frontal and cingulate cerebral areas. Differences between subjects with high (Highs) and low (Lows) level of hypnotizability have been reported, in various tasks, even in the absence of any hypnotic induction and /or specific suggestions [2-5]. In the autonomic domain, our previous studies showed that during simple relaxation, that is relaxation without any specific instruction [6], only Lows decreased their mean heart rate and showed an increased HF spectral component of heart rate variability, in spite of the similar relaxation reported by the two groups. Since EEG spectral analysis revealed an increased gamma power in Highs and a decreased one in Lows, relaxation was considered as a cognitive task modulated by hypnotizability with Highs performing an active cognitive strategy, and Lows responding with the disengagement from any intentional cognitive activity [7].

When not hypnotized Highs receive emotion-laden suggestions, they report a subjective experience corresponding to the suggestion received, i.e fear, and exhibit EEG correlates of activation, but do not show the expected autonomic response, which suggests the possible occurrence of an active buffering of the autonomic output [8]. However, results on hypnotizability related autonomic control are not univocal since other studies have shown that a preeminent sympathetic tone is present in Highs and that medium hypnotizable subjects respond with greater decreases in parasympathetic activity than both Highs and Lows, during emotional stress tasks [9].

The autonomic response to stimulation with different emotional content (positive-negative) and different level of arousal (high-low) has been studied through the use of video clips which allowed identifying autonomic activation patterns specific for various emotional experiences [10-12]. In the present study we used such video clips to evaluate possible hypnotizability-related autonomic responses during cognitive tasks with pleasant or unpleasant content. Thus, Highs and Lows watched video clips in the absence of any specific instruction other than concentrating on the movie. The low attentional load of the movies allowed avoiding possible superimposition between the effects of cognitive load and of the emotional content of the task.

\section{Methods}

Subjects. After their written informed consent following the rules of the Declaration of Helsinki, normal drug-free volunteers (age 19-30) were divided in 2 groups- 20 Highs (10 females) and 26 Lows (15 females)- according to the Stanford Hypnotic Susceptibility Scale, form C [13].

Experimental procedure. Subjects participated in 2 experimental sessions (30 min each) for two low attentional tasks consisting of concentrating on a relaxing 
movie presenting natural scenes associated with soft music (Session R, 46 subjects) or on the triller Shining, by S. Kubrik (Session A, 35 subjects) which was supposed to be arousing. The experimental sessions took place in the morning (9.00-12.00) and were separated by at least 3 days. At the beginning and at the end of each session the participants scored their relaxation (score 010) and, finally, were interviewed about the attention paid to the movie (score 0-10). ECG, respirogram and blood pressure (BP) were recorded and, for off line analysis, sessions were divided into 6 consecutive intervals $(\mathrm{I} 1, \ldots$ I6), 5-minute each.

Data acquisition. ECG was recorded through Red DotTM Ag/AgCl disposable electrodes placed according to the standard DI, while the respirogram (RESP) was obtained through a piezoelectric dc-coupled transducer (Pro-Tech Instruments) wrapped around the chest. ECG was acquired at $1 \mathrm{KHz}$ sampling rate (National Instrument $\mathrm{A} / \mathrm{D}$ converter) and the RR interval series (tachogram) was extracted using a QRS complex detection algorithm based on a threshold derivative method. Blood pressure was monitored through a photopletismograph (Psylab) with a sensor placed on the third phalanx of the middle finger of the left hand and acquired though the same converter used for ECG.

Data analysis. Time domain analysis of RR and blood pressure were performed through mean and standard deviation (SD) values and through the classical $\mathrm{sd} 2$ and sd1 (higher and lower dispersion) dimensions of the Poincaré plot (PP) [14-18], the former being a measure of the Low and Very Low variability (mainly related to sympathetic variability) and the latter an index of the fast variability associated with parasympathetic activity. The mean respiratory frequency (RF) and amplitude (RA) were also evaluated.

Statistical analysis. For session R separate repeated measures ANOVAs were performed on the interview

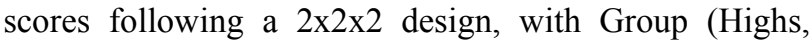
Lows) and Gender (females, males) as Between groups factors and Times (before, after the movie) as Within Group factor. For each autonomic parameter analysis was conducted following a 2 Groups x 2 Genders x 6 Intervals (I1,...I6) design, with Group and Gender as Between groups factors and Intervals as Within Group factor, for session R, and following a 2 Groups x 2 Genders x 5 Intervals $(\mathrm{I} 1, \ldots \mathrm{I}$ ) design for session $\mathrm{A}$ due to numerous missing values in the last interval (I6) of some of the subjects. Furthermore, for session A subjects reporting an increased alertness were grouped and analysed separately from those reporting no change or even relaxation.

\section{Results}

Session R (Table 1.). Interview scores. All subjects felt significantly more relaxed $(F(1,42)=97.197$, $\mathrm{p}<0.0001)$ after the movie than before (mean+SE: before, $5.87 \pm 0.26$; after, 8.26+0.18). Females $(7.93 \pm 0.31)$ reported significantly higher attention to the movie $(\mathrm{F}(1,42)=5.408, \mathrm{p}<0.025)$ than males $(6.87 \pm 0.34)$.

$R R$ and RESP analysis. No significant main effects and interactions were found for mean RR and sd1. SD increased throughout the session, although not significantly $(\mathrm{F}(4,210)=3.334, \quad \mathrm{p}=0.059)$, while $\mathrm{sd} 2$ showed a significant increase across intervals $(\mathrm{F}(5,210)=4.033, \mathrm{p}<0.030)$. A trend toward a Gender effect, with lower mean RR in females than in males, was observed $(\mathrm{F}(1,42)=3.874, \quad \mathrm{p}=0.056)$. No significant change was observed in RF, while RA showed a significant Interval $\mathrm{x}$ Gender $\mathrm{x}$ Group interaction $(\mathrm{F}(5,210)=4.629, \mathrm{p}<0.009)$ corresponding to significant decreases across intervals only in the females belonging to the Highs group $(\mathrm{F}(5,45)=2.369, \mathrm{p}=0.054)$.

$B P$ analysis. No significant main effects were found for $\mathrm{BP}$ mean value. SD showed a significant increase across intervals $(\mathrm{F}(5,210)=3.969, \mathrm{p}<0.011)$ and a significant Interval $\mathrm{x}$ Gender $\mathrm{x}$ Group interaction $(F(5,210)=2.979, p<0.013)$. The latter was sustained by increases of SD across Intervals in Highs, both males and females, $(\mathrm{F}(5,90)=3.231, \mathrm{p}<0.043)$ and in the males of the Lows group (Interval $x$ Gender; $(F(5,120)=4.450$, $\mathrm{p}<0.014)$. Analysis yielded significant Gender $x$ Group $(\mathrm{F}(1,42)=5.982, \mathrm{p}<0.019)$ and Interval $\mathrm{x}$ Gender $\mathrm{x}$ Group interactions $(\mathrm{F}(5,210)=3.559, \mathrm{p}<0.021)$ for $\mathrm{sd} 1$, with a significant increase across intervals in Highs. A Gender effect was also found within Highs $(F(1,18)=6.862$, $\mathrm{p}<0.017)$ with values in females $(1.80 \pm 0.21)$ higher than in males $(1.04 \pm 0.21)$. Significant Interval effect $(\mathrm{F}(5,210)=5.107, \overline{\mathrm{p}}<0.003)$ and Interval $\mathrm{x}$ Gender $\mathrm{x}$ Group interaction $(F(5,210)=2.574, p<0.028)$ were found for $\mathrm{sd} 2$. In particular, $\mathrm{sd} 2$ increased across Intervals in Highs $(\mathrm{F}(5,90)=3.562, \mathrm{p}<0.031)$, while, within Lows, it increased across intervals only in males $(\mathrm{F}(5,50)=4.755$, $\mathrm{p}<0.011$ ).

Session A (Table 1.). Interview scores. Twenty subjects out of 35 reported a significantly increased arousal after watching the movie $(\mathrm{F}(1,16)=40.213$, $\mathrm{p}<0.0001)$. Among the others, a significant Time $x$ Group interaction $((\mathrm{F}(1,9)=14.778, \mathrm{p}<0.04)$ was found, with Highs significantly more relaxed after the movie than before $(\mathrm{F}(1,4)=74.462, \quad \mathrm{p}<0.001)$, while no changes occurred in Lows .

Alerted subjects (Session A-a).

$R R$ and RESP analysis. No significant Interval effect was observed for both mean RR and SD. Analysis of sd 1 $(F(4,64)=3.295, p<0.016)$ yielded significant increases across intervals; sd2 showed tendency to increase across time, in spite of the lack of significant Interval effect or interactions. Both $\mathrm{SD}(\mathrm{F}(1,16)=10.351, \mathrm{p}<0.005)$ and $\mathrm{sd} 2$ $(\mathrm{F}(1,16)=5.396, \mathrm{p}<0.034)$ were significantly higher in 
males than in females during later Intervals. RF and RA did not change across time and did not show any Group effect and interaction.

$B P$ analysis. Mean BP value increased significantly across Intervals $(\mathrm{F}(4,60)=4.017, \mathrm{p}<0.006)$, while no significant change occurred in $\mathrm{SD}, \mathrm{sd} 1, \mathrm{sd} 2$.

\section{Non alerted subjects (Session A-n).}

$R R$ and RESP analysis. No significant effect and interaction were observed for mean RR, SD, sd2, sd1, RF and RA.

$B P$ analysis. Significantly higher values of mean BP were found in females than in males $(\mathrm{F}(1,11)=5.089$, $\mathrm{p}<0.04)$. sd1 exhibited a significant Interval $x$ Gender $x$ Group interaction $(F(4,44)=2.875, p<0.034)$, with higher values in females than in males among Highs during later intervals $(\mathrm{F}(1,5)=8.388, \mathrm{p}<0.034)$ and no differences among Lows.

Table 1. Behaviour of RR and BP in sessions $\mathrm{R}$ and $\mathrm{A}$. $\mathrm{H}=$ Highs, $\mathrm{L}=$ Lows, $\mathrm{F}=$ females, and $\mathrm{M}=$ males.

\begin{tabular}{|c|c|c|c|c|c|c|}
\hline & \multicolumn{2}{|c|}{ Session R } & \multicolumn{2}{|c|}{$\begin{array}{l}\text { Session A- } \mathrm{a} \\
\end{array}$} & \multicolumn{2}{|c|}{ Session A-n } \\
\hline & $\mathrm{RR}$ & BPmean & $\mathrm{RR}$ & BPmean & $\mathrm{RR}$ & BPmean \\
\hline mean value & & & & $\mathbf{T}$ & & \\
\hline SD & & † $\quad \mathrm{H}$ & $\mathbf{T}$ & & & \\
\hline $\operatorname{sd} 2$ & $\mathbf{T}$ & $\begin{array}{r}\mathrm{H} \\
\mathrm{ML}\end{array}$ & $\mathbf{T}$ & & & \\
\hline sd1 & & $\mathrm{H}$ & $\mathbf{1}$ & & & $\mathbf{1}$ \\
\hline
\end{tabular}

\section{Discussion and conclusions}

The present findings show that during a low attentional, relaxing task both Highs and Lows do not modify their mean heart rate, in spite of an increase in sd2, indicating modifications of the slow HRV components. This is at variance with what previously found during simple relaxation sessions [7] when no attentional performance was required and the High Frequency, parasympathetic component of RR variability, was responsible for the slight $R R$ increases observed in Lows across time.

Hypnotizability related differences emerge in mean blood pressure whose changes in variability across time indicate that, during the relaxing movie, the variability expressed by sd2 (mainly related to sympathetic activity) increased in both groups, although only in males among Lows, while the parasympathetic variability increased only in Highs, which might be in line with the hypothesized favourable role of hypnotic susceptibility [19] in cardiovascular health. The results concerning the participants not aroused by the alerting movie (Session A-n) were similar to those obtained during the relaxing movie. It is noticeable that, among these participants, Highs reported an increased relaxation in spite of the arousing movie, while in Lows it was simply not effective. However, the autonomic changes of Highs during Session A-n were limited to females whose subjective autonomic monitoring seems to be more reliable than the males one. This is at variance with Session R in which Highs autonomic changes were independent of gender. All the participants reporting a decreased relaxation during the alerting movie (Session A-a) showed increases in both the sympathetic and parasympathetic variability of RR with no change in its mean value, which confirms the results obtained during the guided imagery of an unpleasant situation [8], mental stress $[20,21]$ and experimental pain [22]. At variance, mean blood pressure increased. This might indicate a hypnotizability-dependent different interaction between the sympathetic and the endotelial control of vascular resistance during different tasks. In fact, the endothelial control did not change during painful stimulation and mental computation in Highs [20-22], while the arousing movie, which increased mean blood pressure in the absence of changes in heart rate, actually induced changes in the peripheral resistance also in Highs.

The present findings show differences in the hypnotizability-related modulation of heart rate and mean blood pressure variability, which is in line with other authors' results reporting hemodynamic responses to stress involving mainly the cardiac output or peripheral resistances [23] and prompts further investigation on the possibly different effects of various cognitive tasks at cardiac and vascular level. In line with previous studies [20-22], the present results suggest, for hypnotizability, a greater role in the control of the vascular resistance than in heart rate modulation. In addition, they highlight the hypnotizability-related effect of the emotional content associated with a relaxation response.

A limitation of the study is due to the low number of subjects really aroused by the arousing task, which makes the suppression of the gender differences in the mean blood pressure control observed during the arousing task unreliable.

Finally, we would like to remark that the concurrent increases in the sympathetic and parasympathetic activities observed during the relaxing task as well as during the successfully activating one support the view of a wide modulability of the autonomic space not limited to balance mechanisms [24].

\section{References}

[1] Green JP, Barabasz AF, Barrett D, Montgomery GH Forging ahead: the 2003 APA Division 30 definition of hypnosis. Int J Clin Exp Hypn 2005;53: 259-64

[2] Carli G, Manzoni D, Santarcangelo EL. Hypnotizabilityrelated integration of perception and action. Cogn Neuropsychol 2008;in press

[3] Santarcangelo EL, Balocchi R, Scattina E, Manzoni D, Bruschini L, Ghelarducci B, Varanini M. Hypnotizability- 
dependent modulation of the changes in heart rate control induced by upright stance. Brain Res Bull 2008;75:692-97.

[4] Santarcangelo EL, Scattina E, Orsini P, Bruschini, Ghelarducci B, Manzoni D. Effects of vestibular and neck proprioceptive stimulation on posture as a function of hypnotizability. Int J Clin Exp Hypn 2008;56:170-84.

[5] Santarcangelo EL , Scattina E, Carli G, Macerata A, Manzoni D Hypnotizability-dependent modulation of postural control: effects of the alteration of the visual and leg proprioceptive input. Exp Brain Res 2008;in press.

[6] Benson H, Arns PA, Hoffman JW. The relaxation response and hypnosis. Int J Clin Exp Hypn 1981;29: 259-270.

[7] Sebastiani L, Simoni A, Gemignani A, Ghelarducci B, Santarcangelo EL. Relaxation as a cognitive task. Arch Ital Biol 2005;143:1-12.

[8] Sebastiani L, Simoni A, Gemignani A, Ghelarducci B, Santarcangelo EL. Human hypnosis: autonomic and electroencephalographic correlates of a guided multimodal cognitive-emotional imagery. Neurosci Lett 2003;338:414.

[9] Jørgensen MM, Zacharia R.Autonomic reactivity to cognitive and emotional stress of low, medium, and high hypnotizable healthy subjects: testing predictions from the high risk model of threat perception. Int J Clin Exp Hypn 2002;50:248-75.

[10] Rainville P, Bechara A, Naqvi N, Damasio AR. Basic emotions are associated with distinct patterns of cardiorespiratory activity.Int J Psychophisiol 2006;61:5-18.

[11] Frazier TW, Strauss ME, Steinhauer SR. Respiratory Sinus Arrhythmia as an index of emotional response in toung adults. Psychophysiol 2004;41:75-83.

[12] Gomez P, Zimmermann P, Guttormsen-Schar S, Danuser B. Respiratory responses associated with affective processing of film stimuli. Biol Psychol 2004;68:223-35.

[13] Weitzenhoffer AM, Hilgard, E.R. Stanford Hypnotic Susceptibility Scale, form C. Consulting Psychologist Press 1962;Palo Alto, CA.

[14] Toichi M, Kamio Y. Paradoxical autonomic response to mental task in autism. J Autism Dev Disord 2003;33:41726.

[15] Toichi M, Kubota Y, Murai T, Kamio Y, Sakihama M, Toriuchi T, Inakuma T, Sengoku A, Miyoshi K. The influence of psychotic states on the autonomic nervous system in schizophrenia. Int J Psychophysiol 1999;31:14754.
[16] Toichi M, Sugiura T, Murai T, Sengoku A. A new method of assessing cardiac autonomic function and its comparison with spectral analysis and coefficient of variation of R-R interval. J Auton Nerv Syst 1997;62:79-84.

[17] Kubota Y, Sato W, Toichi M, Murai T, Okada T, Hayashi A, Sengoku A. Frontal midline theta rhythm is correlated with cardiac autonomic activities during the performance of an attention demanding meditation procedure. Cogn Brain Res 2001;11:281-87.

[18] Balocchi R, Cantini F, Varanini M, Raimondi G, Legramante JM, Macerata A. Revisiting the potentials of time-domain indexes in the short-term HRV analysis. Biomed Technol 2006;51:190-93.

[19] Santarcangelo EL, Sebastiani L. Hypnotizability as an adaptive trait. Contemporary Hypnosis 2004;21:3-13

[20] Jambrik Z, Santarcangelo EL, Ghelarducci B, Picano E, Sebastiani L. Does hypnotisability modulate the stressrelated endothelial dysfunction. Brain Res Bull 2004;63: 213-21.

[21] Jambrik Z, Sebastiani L, Picano E, Ghelarducci B, Santarcangelo EL. Hypnotic modulation of flow-mediated endothelial response to mental stress. Int J Psychophysiol 2005a;55: 221-227.

[22] Jambrik Z, Carli G, Rudish T, Varga A, Forster T, Santarcangelo EL. Modulation of pain-induced endothelial dysfunction by hypnotisability. Pain 2005b;116:181-86.

[23] Nogawa M, Yamakoshi T, Ikarashi A, Tanaka S, Yamakoshi K. Assessment of stress-induced hemodynamic responses using multipurpose non-invasive continuous cardiovascular monitoring system. Conf Proc IEEE Eng Med Biol Soc 2006;6537-9.

[24] Berntson GG, Cacioppo JT, Quiqley KS. Cardiac psychophysiology and autonomic space in humans: empirical perspectives and conceptual implications.Psychol Bull 1993;114: 296-322.

Address for correspondence:

Rita Balocchi

Istituto di Fisiologia Clinica

CNR-Area della Ricerca

Via Moruzzi, 56124-Pisa, Italy

E-mail address: balocchi@ifc.cnr.it 